\title{
Steps towards modern trends in district heating
}

\author{
Lubomir Vasek ${ }^{l}$ and Viliam Dolinay ${ }^{1, a}$ \\ ${ }^{1}$ Tomas Bata University in Zlín, Faculty of Applied Informatics, Nad Stranemi 4511, 76005 Zlin, Czech Republic
}

\begin{abstract}
This paper focuses on new trends in district heating a cooling (DHC) area and algorithms allowing incorporating new technologies and performing optimal control. Classical district heating usually means huge source (as heating plant) and set of pipes which transfer heat energy through a medium, mostly water, across whole town and chilled water is returning back to the plant. Let's imagine a modern city where buildings are consuming only a fraction of the energy contrary to what buildings required in the past. And especially during sunny or windy days, they have energy to spare. Around of such modern city is not only the one big heating plant, but perhaps solar and wind farms, waste incinerators, industrial companies with energy surpluses. Simply in this modern city are dozens, perhaps hundreds of small energy producers that share pipe network or at least part of it. In such a district energy system, production planning is more difficult. And not only production, modern houses with minimal heat loss and data connections also allow to plan consumption more effectively. The aim is to achieve the best solution evaluated by the objective function, usually determined by minimizing the production and distribution costs and providing meets the needs of energy consumers. The method presented in this paper is based on a simulation using the proposed holonic distributed model. This model also introduces the idea of general prosumers strategy, where all active elements within the modern DHC system are represented by prosumer objects. The prosumers are perceived as objects able to actively participate in the planning and realization of the production and consumption of energy. It is assumed that the general behaviour of the object in DHC is the same, no matter how they differ in size and design. Thus, all the objects are defined by two characteristics - the ability to produce and consume. The model based on this basic principle, of course, with the most accurate information about the particular values at a time, object properties and other, should provide tools for simulation and control of modern DHC. In a broader perspective model can be applied to superior units such Smart Energy Grids - understood as a system integrating electricity (Smart Grids) and heat and cool (Smart Thermal Grids) features.
\end{abstract}

\section{Introduction}

Modern civilization is aware that energy resources are limited and at the same time this civilization requires greater comfort and convenience, which are often more energy-demanding. Intensely are therefore addressed issues of sustainability and efficiency. In the field of energetics, it means to lower consumption of primary energy. The main obstacles to these goals are mainly technical capabilities and the economic performances of individual countries [1]. However, if the countries want to behave ecologically and foresight into the future, they must seek these goals.

An effort to energy saving also means the introduction of new principles and technologies. In the area of district heating and cooling variety of producers grows, smaller and usually more ecological sources are involved, for example geothermal, solar, wind sources, industrial waste heat recovery, etc. [2-4]. The role of consumers is also more important. The expected benefits can be achieved only by mutual cooperation of all DHC elements [5,6]. Upgraded and improved technologies require support from new and intelligent approaches in control. Nevertheless, the modern concept of control cannot be understood only as an algorithm implemented in a specific object, but the entire hierarchy in which objects collaborate to achieve common goals. Even though the elements of the system are directed towards a common goal such a large and often disparate systems as DHC is not appropriate to centralize. It seems better to distribute the competence and ensure cooperation. Distributed control approaches in the proposed model are represented by holonic structure (multi-agent), which is looking for the optimal solution of the objective function.

\section{Generations of District Heating}

District heating is likely the most efficient, fuel-flexible and environmentally friendly method to produce and distribute energy on the market. It is sustainable, affordable and community-based. On a larger scale district heating system began to develop as early as the 19th century. Since then, the technology and materials gradually improved also DH somehow changed its face. We can talk about generations of $\mathrm{DH}$.

\footnotetext{
a Corresponding author: vdolinay@fai.utb.cz
} 


\subsection{Previous generation of $\mathrm{DH}$}

The first generation of district heating systems used steam as the heat carrier. These systems were first introduced in USA in the $1880 \mathrm{~s}$. Almost all district heating systems established until 1930 used this technology, both in USA and Europe. Typical components were steam pipes in concrete ducts, steam traps, and compensators.

The second generation of systems used pressurized hot water as the heat carrier, with supply temperatures mostly over $100{ }^{\circ} \mathrm{C}$. These systems emerged in the $1930 \mathrm{~s}$ and dominated all new systems until the 1970s. Typical components were water pipes in concrete ducts, large tube-and-shell heat exchangers, and material-intensive, large, and heavy valves.

The third generation of systems was introduced in the 1970s and took a major share of all extensions in the 1980s and beyond. Pressurized water is still the heat carrier, but the supply temperatures are often below 100 ${ }^{\circ} \mathrm{C}$. This third generation is sometimes referred to as "Scandinavian district heating technology", since many district heating component manufacturers are Scandinavian. Typical components are prefabricated, preinsulated pipes directly buried into the ground, compact substations using plate stainless steel heat exchangers, and material lean components [5].

\subsection{The 4th Generation District Heating}

The future 4th Generation District Heating (4GDH) system is defined as a coherent technological and institutional concept, which by means of smart thermal grids assists the appropriate development of sustainable energy systems [5]. 4GDH systems provide the heat supply of low-energy buildings with low grid losses in a way in which the use of low-temperature heat sources is integrated with the operation of smart energy systems. See Figure 1. The concept involves the development of an institutional and organizational framework to facilitate suitable cost and motivation structures [7].

In the context of the 4th generations, we can also talk about Smart Thermal Grids or in combination with electric power is also used term Smart Energy Grid.

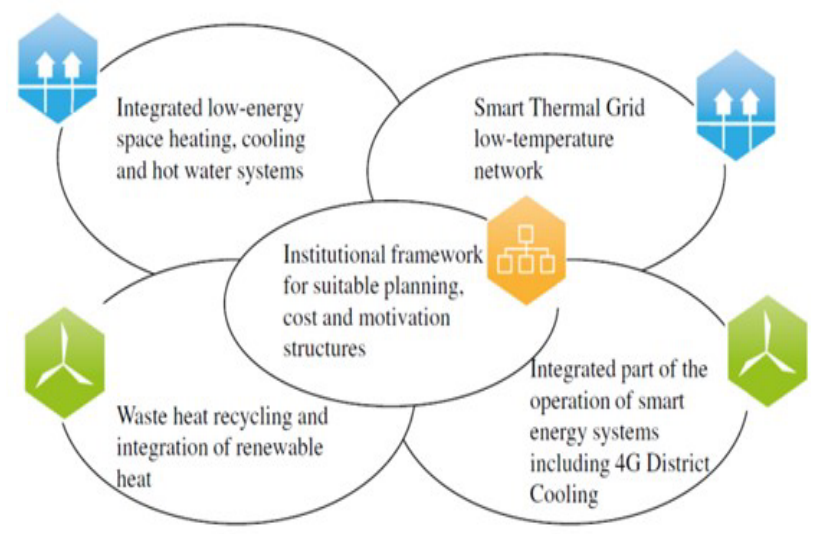

Fig. 1. Illustration of the concept of $4 \mathrm{GDH}$ including smart thermal grids [4].

\subsection{The coming of the fifth generation}

Even though the 4th generation is still called future generation and implementation only began in some large cities and many sources estimate that the main era for fourth generation will start in 2020 [13], especially the rapid development of digitization and mobile technology suggests the following directions - the features of the fifth generation [14].

\section{Deployment of the $4^{\text {th }}$ and $5^{\text {th }}$ generation ideas into practice}

The application of ideas of the 4th generation (especially rebuilding networks and building new sources) will be quite slow in the conditions of the Czech Republic (author's home country). District heating is not too prepared for this, and the investments needed to build, for example a diverse structure of smaller sources and their interconnection, will be huge and build it will take many years. Instead, the implementation of digital technologies (the character of the 5th generation) is closer, so it seems that rise of these generations will not be consecutive but concurrent.

However, a number of developmental steps can be started already. The areas of development and use of the developed means can be divided into the following three areas - heat energy production, distribution and consumption. In each of these areas, it is possible to specify the objectives and research and development activities from two areas - technical-technological and economic. Of course, both of these areas are very closely related, but it will be appropriate to use separate but closely interrelated models - technical-technological and economic.

The objectives can be formulated as follows (including the design of individual steps and stages of the solution):

\subsection{Heat energy production area}

\subsubsection{Technical-technological objectives}

a) Changes in the technological elements in DHCS.

b) Including a whole range of other possible and usable heat sources in DHC. These are different types of renewable sources (solar, wind, geothermal), waste heat from incineration plants, technological and manufacturing processes, surplus electricity in the grid and, last but not least, the thermal energy accumulators that can be used in certain phases of their activity. A special case is incorporating the thermal energy accumulators as possible sources (from the modelling point of view).

c) Adjustments to the control system of individual technological elements

d) Predictor of disposable production capacity of individual sources depending on production factors and time. The production capacities of each source are classified by priority into several categories. The use of individual sources should 
be managed by their immediate effectiveness and will therefore depend on a number of influencing factors and will be time-varying. So we can talk about a system where the significant characteristics of their individual elements change over time. To model these dependencies, individual sources can be characterized in priorities that express their efficiency in obtaining thermal energy. Under this priority, resources can be categorized into the following categories:

Prio 1 ("necessary") - "residual heat" from cogeneration power generation

Prio 2 ("appropriate") - RES in case of appropriate (eg climatic) conditions

Prio 3 ("normal") - production under normal conditions

Prio 4 ("emergency") - production under critical conditions

For each category, the economic model will generally consider the different cost (production costs) of the heat produced:

\subsubsection{Economic objectives}

Optimizing the load of individual production sources in connection with the optimization of energy flows within the distribution of thermal energy

\subsection{Area of heat energy distribution}

\subsubsection{Technical-technological objectives}

Changes in the technological elements - distribution part of DHCS.

\subsubsection{Economic objectives}

Optimization of energy flows using proposed predictor of the disposable production capacities of the individual sources and heat consumption for individual types of consumers.

\subsection{Heat consumption area}

\subsubsection{Technical-technological objectives}

a) Creating an intelligent heat consumption predictor for individual types of consumers (or individual consumers) that will be based on an analysis of historical data to determine heat consumption dependencies on relevant factors: - Time characteristics of consumption - actual values, historical trends in the previous period, trends in the analogous historical period (similar days or longer time periods), average values for analogous periods - Type of heating operation - daily, weekly, seasonal

- Significant climate conditions (external temperature, sunshine, wind, etc.), their time characteristics, their predicted values from meteorological forecasts

b) Incorporating the heat accumulator as an consumer into the DHCS

c) The output is a time-space list of heat consumption requirements for individual consumers that will be used as input to the distribution subsystem

\subsubsection{Economic objectives}

Analysis of the possibilities and benefits of economic motivation of consumers for the timevarying consumption of heat energy.

This contribution, which follows up on the long-term research focused on the technical-technological area, specifically on prediction of heat consumption in DHC, focuses on a more comprehensive view of the whole DHC system, i.e. its individual parts and their interaction. So we can talk about an integrative view of DHC.

\section{DHC model}

Having the ability to effectively control such a complex system as the modern DHC requires having its model available to test (simulate) a number of situations in virtual conditions.

Such a model was introduced in [10] and thus only a reminder of basic terms will follow.

The proposed model is multi-agent (holonic) working with the idea of prosumer concept.

\subsection{Holonic system}

The holonic system can be understood as a specific implementation of multi-agent concept. Holons (agent type), could be defined as autonomous and co-operative building blocks of the system for transforming, transporting, storing and/or validating information and physical objects. The holon consists of an information processing part and often a physical processing part. A holon can be part of another holon. It is also possible to see it as a model of a particular element, i.e. part of the model of the entire system. In this sense is holon used in this article.

The internal structure of holons can be made up of a group of other holons, which can be described as "subholons". Any such subholon is, of course, full holon. This allows a very flexible way to define entire holonic system.

The most important features of holon are autonomy and co-operation. Autonomy is characterized by its ability of self-regulation, i.e. the capability to apply the flexible strategy which allows holon to respond differently to changes in its relevant environment [5]. Set of holons including their mutual relations is referred as holarchy. Holarchy is a system of holons that can cooperate to achieve a goal or objective. The holarchy defines the basic rules for co-operation of the holons and thereby limits their autonomy. 


\subsection{Prosumers concept}

For the purpose of modelling processes in DHC in the production, distribution and consumption of heat presented in this article can be as prosumer identified as an element which acts as an energy source or(and) as a consumer. The prosumer can act on both roles, for example a heat accumulator that consumes energy when it is charging, and can be understood as a source when discharged. So the prosumer has a component of production and a component of consumption. However, any of them may be zero. Therefore prosumer, at least in the context of this article, will be used to denote all active elements in DHC.

\section{DHC model structure}

The proposed model of DHC consist of two fundamental components - set of prosumer and distribution network. The characteristic attributes which specify properties relevant for the modelling and analysis of DHC behaviour are defined for each element.

Description of such modelled system is shown in Figure 2 .

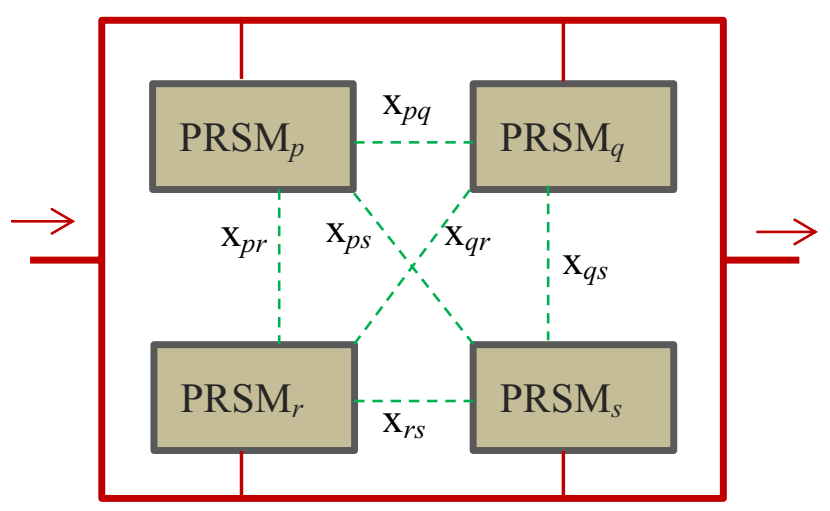

Fig. 2. A schematic description of part of DHC network topology

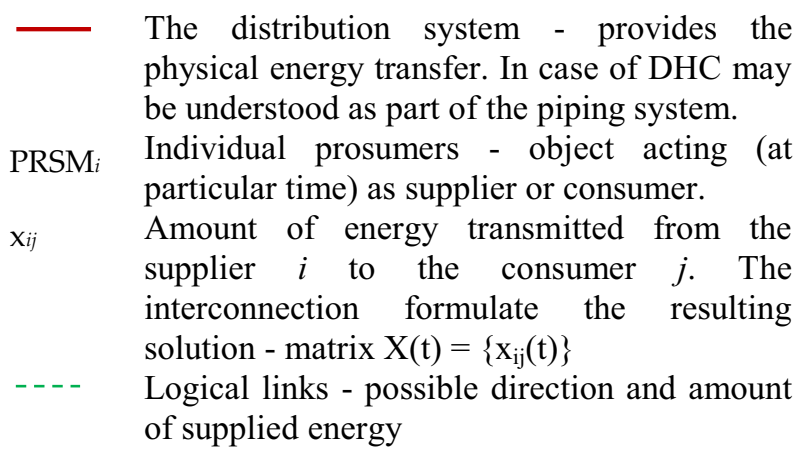

\subsection{Prosumer}

Each prosumer $\mathrm{PRSM}_{\mathrm{i}}$ is characterized in relation to the analysed processes of production, distribution and consumption of heat with a number of attributes that can be divided into two groups - producer attributes and consumer attributes. Most of these attributes are function of time.

1. Producer attributes - for $\mathrm{PRSM}_{\mathrm{i}}$ it is necessary to know the following:

a. Performance data for each source as a function of time $t$ and any other parameters, referred to as a vector $P P$ (production parameters). They are specific for the process of heat production in a given source and affect produced

b. Price data of the supplied energy for each source, again as a function of time $t$ and any other parameters referred to as a vector $P P$

The following figure illustrates the potential cost dependence on the produced amount. The costs are the lowest (optimal) if production is also within the optimum interval $<\mathrm{P}_{\text {opt } 1}, \mathrm{P}_{\text {opt } 2}>$. A production below (in interval $<\mathrm{P}_{\min }, \mathrm{P}_{\text {opt } 1}>$ ) or above (in interval $<\mathrm{P}_{\text {opt } 2 \text {, }}$ $\mathrm{P}_{\max }>$ ) the optimum means increased costs to produce a "unit of energy". In case that the curve will not be known throughout the interval, it can be approximated - for example the mean value for each interval is applied. It is of course also possible to apply various approximations, e.g., linear see orange dashed line in the figure.

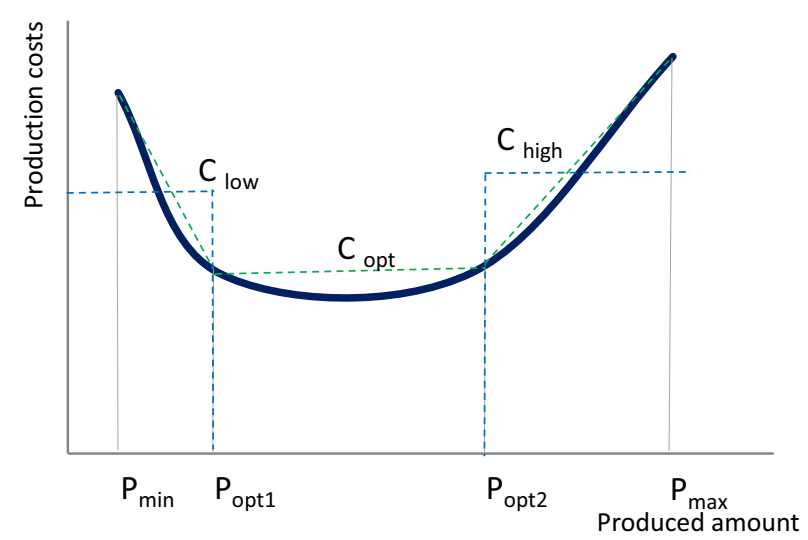

Fig. 3. The dependence of cost on the produced quantity

\section{Consumer attributes}

Data on the required consumption of each consumer as a function of time $t$ and any other parameters $C P$

\subsection{Distribution network}

Another important part of DHC and therefore its model is distribution network. It connects individual prosumers and allows the transfer of thermal energy between them in a desired direction and time varying quantities. The distribution network is characterized by a topology which is generally very stable and varies, if at all, only in very specific points in time - the physical reconstruction of the network, connecting or disconnecting certain prosumer etc. 


\section{Optimization of energy flow in DHC}

Model specified previously is used to find the optimal distribution of energy flows between the individual prosumers to satisfy their consumption demands and apply the most efficient use of production capacity of each prosumer (as produced). There should be highlighted that the solutions are being sought for dynamic system, i.e. these energy flows are changing over time. Result (solution) is captured in the matrix X(t) $=\left\{\mathrm{x}_{\mathrm{ij}}(\mathrm{t})\right\}$, which has a similar structure as the matrix $\mathrm{R}$. The value of $x_{i j}(t)$ is the amount of energy transmitted from the supplier to the consumer $j$ (at time $\mathrm{t}$ ) $\left(\mathrm{x}_{\mathrm{ij}}(\mathrm{t})>=0\right.$ ).

The solution will satisfy the condition of minimum objective function. That is, in this case the value of the total cost $\mathrm{CF}$ which is calculated as sum of cost $\mathrm{CP}$ to produce and the cost $\mathrm{CT}$ to transfer whole needed amount of heat energy between prosumers for the entire time period of heating, thus

$$
C F=\min (C P+C T)
$$

If the time interval $\mathrm{TI}$ is divided into $\mathrm{M}$ particular time intervals identified as $\Delta t_{k}=t_{k+1}-t_{k}, k=1,2, \ldots, M$, the equation for the production costs is

$$
C P=\sum_{k=1}^{M}\left(\sum_{i=1}^{N} \sum_{j=1}^{N} x_{i j}(t) * c_{i}(t)\right) \Delta t_{k}
$$

here $c_{i}(t)$ are costs (the cost of one unit of heat energy producing in source (prosumer) $i$ in the corresponding time interval $\Delta t_{k}$.

Similarly, for transportation (distribution) is obtained equation

$$
C T=\sum_{\mathrm{k}=1}^{\mathrm{M}}\left(\sum_{i=1}^{m} \sum_{j=1}^{n} x_{i j}(t) * c t_{l}(t)\right) \Delta t_{k}
$$

where $c d_{l}(t)$ are the cost of the transport of energy unit through route $l$ - their tag (index) is stored in matrix R.

Obtained solutions X must fulfil a number of conditions, see [10]

To solve such tasks usually means to define a function (target or purpose function). Very often this function is defined in economic terms as a cost function that calculates the necessary cost for given solution. A solution is determined by set of, mostly integer, parameters which characterize the process. If the values of parameters are such, that the restrictive conditions come true, the solution is defined as feasible solution. It is usually possible to find it by a heuristic method. However, very difficult is to find the optimal solution. Such solution is characterized in that the selected target function for it acquires its most extreme, usually minimum value.

Theoretically, it is easy to find the optimal solution by calculating all the possible variants and determine if it is a feasible solution. For feasible solution calculate the value of the target function and choose the one in which reaches the target function extreme value - it is so called the Brute Force algorithm. It is clear, however for the larger scheduling task the amount of possible variants is huge and this method is not due time requirements practically applicable.

In our case, when the parameters (transferred thermal power or amount of heat energy) are not integer values, the situation is more complicated becausethe number of possible variants can grow beyond all bounds. Thereafter the only possible way is to find a suitable procedure based on the heuristic methods and tries to find in reasonable time the solution, which is good enough, because there is in general unfortunately not guarantee finding the optimal one.

\subsection{Methods}

Searching the best variant of scheduling energy flows will be designed as a suitable procedure based on the set of heuristic methods. Methods will be developed to explore feasible solution. Such methods may vary either by using different algorithms, or, in case that the used algorithms are parametrized, only vary the values of those parameters. The search process then proceed in a way that successively, by a suitable mechanism, will individual heuristics methods propose their solutions and the best result will be sought - evaluation will be performed using the chosen target function. The mechanism to prepare sequence of used methods can be established on the basis of the results obtained previously and of course, it can interfere with human - the dispatcher. The search progress will be stopped after a predetermined time and selected the best of the calculated variants.

\section{Conclusions}

New trends in the field of heating and cooling systems were analyzed, built the mathematical description of the problem and prepared the first proposal of the model. The fundamental ideas of this model and algorithms to seek optimal flow of energy in modern DHC systems were presented. Since finding the optimal result is a rather complicated problem, for the solution were selected heuristic methods. They were introduced, but currently need to be more tested and verified. However, the ideas presented in this paper mostly focus the future arrangement of district heating, and also for this reason it is more or less solved in the laboratory (simulated) conditions. It is however assumed that at least the component parts will be soon confronted with real data from selected parts of the real district heating.

\section{Acknowledgment}

This work was supported by the Ministry of Education, Youth and Sports of the Czech Republic within the National Sustainability Programme project No. LO1303 (MSMT-7778/2014). 


\section{References}

1. 4DH, 4GDH Definition, Available from: $<$ http://www.4dh.dk/about-4dh/4gdh-definition>

2. European Commission, Energy Strategy, Available from: <http://ec.europa.eu/energy/en/topics/energystrategy>

3. A. Giret, V. Botti, In Journal of Intelligent Manufacturing, p. 645-659 (2004)

4. H. Lund, S. Werner, R. Wiltshire, S. Svendsen, J. E. Thorsen, F. Hvelplund, B. V. Mathiesen, 4th Generation District Heating (4GDH): Integrating smart thermal grids into future sustainable energy systems, Energy, 68, (2014),

5. L. Vasek, V. Dolinay, In Recent Advances in Systems. New Jersey, Piscataway, p. 559-563, (2015)

6. P. Navratil, J. Klapka, J. Balate, P. Konecny, In: Proceedings of the 18th International Conference on Soft Computing MENDEL, p. 356-362, (2012)

7. M. T. Tsaya, W. M. Linb, Elect. Power Energy Syst., 22, p. 367-373, (2000)

8. C. Johansson, F. Wernstedt, P. Davidsson, Deployment of Agent Based Load Control in District Heating Systems. First International Workshop on Agent Technologies for Energy Systems, (2010)

9. S. Fawkes, The Rise of the Energy Prosumer, Available from: <http://www.heatconference.co.uk/ index.php/blog/entry/the-rise-of-the-energyprosumer $>(2015)$

10. L. Vasek, V. Dolinay, Holonic Model of DHC for Energy Flow Optimization. In IFAC-PapersOnline. Laxenburg, 2016, p. 413-418, (2016)

11. D. Janacova, H. Charvatova, K. Kolomazník, V Vasek, P. Mokrejs, R. Drga, In International Journal of Mathematical Models and Methods in Applied Sciences, 5, p. 1094-1101, (2011)

12. J. Novak, P. Chalupa, V. Bobal, In IEEE Transactions on Power Systems, p. 2450-2459, 4 (2011)

13. C40 \& UNEP, Good Practice Guide, In District Energy, (2016)

14. M. Kalli, Fifth Generation of District Heating Energy Solutiion for Future Smart Cities, In Euro Heat \& Power, p $24-26,2$ (2017) 